\title{
Characteristics of arcs between porous carbon electrodes
}

\author{
E. CARVOU, J.L. Le GARREC, J. B.A. MITCHELL
}

Institut de Physique de Rennes, UMR du CNRS N 6251, Université de Rennes 1, Rennes, France,

\begin{abstract}
Arcs between carbon electrodes present some specific differences compared to metallic arcs. The arc voltage is higher, but does not attain a stable value displaying large fluctuations. Indeed, the arcs are produced by the direct sublimation of the electrodes, without passing through a molten phase. The arc production is also facilitated both by circuit breaking or electric field breakdown. In this work, arcing has been examined under various conditions (voltage, current, opening or fixed inter-electrode gap) and particular attention has been given to the ability of the system to sustain the arc due to re-ignition. Small Angle X-Ray Scattering has been used to examine the formation of particles within carbon arcs and again the results are different from what is found with metallic electrodes, the particles being larger with rougher surfaces. The ultimate aim of this study was to gain new knowledge concerning these arcs, and their consequences for electrical safety (arc faults in wires).
\end{abstract}

\section{Introduction}

Electrical fires due to degraded wiring are a major source of personal injury and property damage [1]. Efforts have been made to understand the development of 
cable faults in order to devise means of predicting problems before the onset of catastrophic arcing leads to an uncontrolled ignition phenomenon [1-7]. When a multi-conductor cable is cut so that adjacent metal wires with differential voltages come into too close contact, this can lead to electrical sparking that eventually develops into a full blown arc. The very high temperature in the arc $\left(>6000^{\circ} \mathrm{C}\right)$ and the subsequent violent release of burning vapor (Figure 1 and video 1 of supplemental material) can set fire to flammable material in the vicinity of the damaged cable.

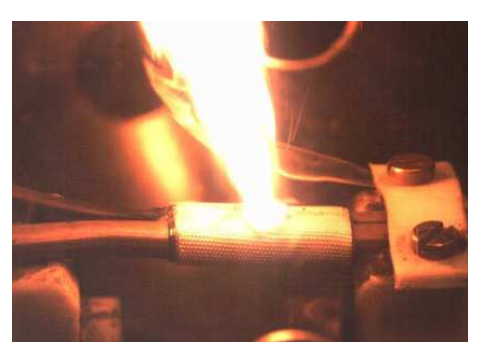

Fig. 1: A two-wire cable previously damaged by sparking, catches fire with the application of $220 \mathrm{~V}$ and $8 \mathrm{~A}$. The picture is taken from a slow-motion video (Supplemental material, video 1 ). 
Another source of arcing occurs when the insulation of a cable is damaged and charring leads to the formation of pockets of carbonized material. Sparking and arc formation can then develop between these pockets of material if they are in contact with the metal conductors (Figure 2 and supplemental material, video 2 ).

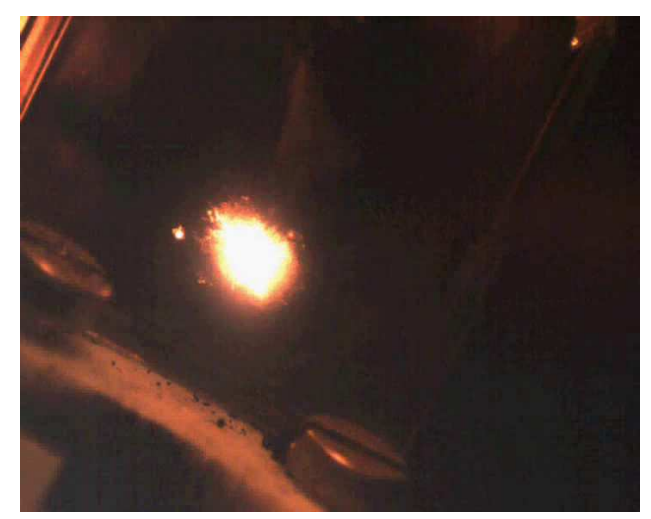

Fig. 2: Glowing pockets of hot carbon within the insulation of a damaged two-wire cable that lead to arc re-ignition. Picture taken from a slow motion video (Supplemental material, video 2).

If the wire is carrying an alternating (AC) current, then the voltage between the adjacent, electrically polarized components (adjacent wires, carbonized pockets) varies from zero to a maximum and back to zero again during a half-cycle. This may lead to the extinction of the arc at the end of the half-cycle but if an arc can reignite at each current half-cycle, then it is more likely for a fire to occur $[1,2,4,7]$. We have been interested in determining the likelihood of the occurrence of this re-ignition phenomenon and so have performed a series of experiments to observe arcing using different electrode materials with various separations and electrical characteristics. We have found that arcing between carbon electrodes is much easier to achieve than with metallic electrodes and this has significant consequences with respect to cable degradation. We have 
also used synchrotron radiation to explore the formation of particles in a free burning carbon arc and the implications of these results to the present problem will be presented.

Two arc ignition configurations have been studied. The first was where the electrodes, initially in contact, were quickly opened to a pre-set inter-electrode gap. This is conventionally referred to as contact breaking. In the second, the electrodes were separated prior to the application of the voltage and the gap length was maintained constant during the tests. These two conditions represent the cases where the arcing occurs with and without the presence of material contact. For the breaking arc tests, conditions under which the arc could reignite were determined. Indeed, this is influenced by the current, electrode gap and electrode material. It is found that, for an $\mathrm{AC}$ arc current of $4 \mathrm{~A}$ and for a $300 \mu \mathrm{m}$ gap, the arc could indeed always reignite. For fixed open gap arc ignition, in addition to the influence of the current and gap, we have also studied the influence of electrode material (graphite or copper, mixed electrodes). In these studies, measurements were made of the voltage required for arc ignition and the subsequent voltage maintained across the arc (which presents a resistive path between the electrodes).

\section{Experimental setup and procedure}

The experimental apparatus is a fully automated device, developed from a DC apparatus described in previous papers [8]. It has been modified to perform contact breaking under alternating current with $220 \mathrm{~V}_{\mathrm{rms}}$ and a current from 0.5 to $10 \mathrm{~A}_{\mathrm{rms}}$. It is mainly composed of an electrical part (power supply, variable resistance) and a mechanical part using a stepping motor coupled with a sliding plate, which ensures the 
controlled separation between the fixed and the moving contact.

A digital oscilloscope with a $500 \mathrm{MHz}$ probe, samples and stores the voltage characteristics of the arc during the break, i.e. Va(t) and Ia(t). A computer, equipped with Testpoint software, is used to control the experimental procedure, the power supply, motor, data collection and analysis.

\subsection{AC Breaking Arc}

In this experiment, the contacts were made from porous graphite (PG-60: National Electrical Carbon Products, Inc., porosity, $48 \%$, average pore diameter $33 \mu \mathrm{m})$. The 4 mm diameter moving electrode had a rounded facing surface while the fixed electrode of the same diameter had a flat surface. This allows us to have a more precise control of the inter-electrode distance (no problems of parallelism) and to have a more localized arc. The arcs were initiated by breaking the contact. The purpose of the experiment was to determine the influence of the final inter-electrode gap and the current on the capability of the arc to maintain itself at each half-cycle.

In order to have an accurate a measurement of the inter-electrode gap when arcing appears and hence of the overall arc length, the final point of electrical contact between electrodes, just before the contact opened, was determined. This was done by first applying a voltage of $5 \mathrm{~V}$ DC to the circuit which is too low to induce arcing. The initially closed electrodes were then moved apart as the voltage between them was monitored. When the electrodes are separated, the measured voltage attains the supply voltage $(5 \mathrm{~V})$. The electrodes are then moved back until the measured voltage is seen to drops as current begins to flow in the circuit. (This voltage is not zero as the contacts have their proper resistance between them). This position is taken as the final point of 
contact. The 220 VAC was then applied and the electrodes separated to the desired gap at an opening speed of $0.02 \mathrm{~m} / \mathrm{s}$. This rapid opening allowed a stable arc to be formed. It was found that with graphite electrodes, arcs were then always observed to reignite at each half-cycle with a current of $4 \mathrm{~A}$ and an electrode separation of $100 \mu \mathrm{m}$. These arcs are manifest not only visually but also by the presence of an "arc voltage" measured between the open electrodes with a value of about $20 \mathrm{~V}$ at this gap (Fig. 3). It is also seen that this voltage is very "noisy" due to the chaotic nature of the arc which is generated from a rapidly moving cathode spot.

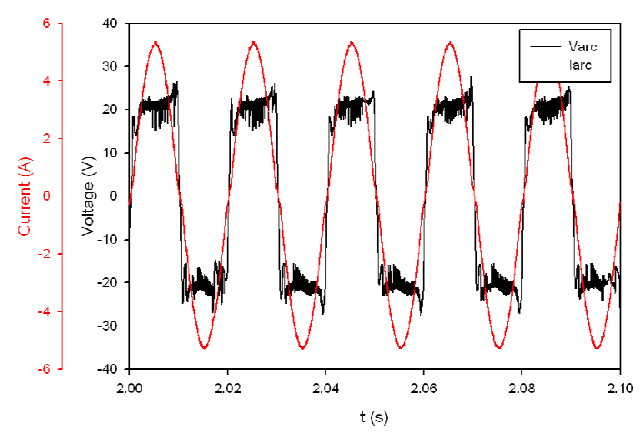

Fig. 3 Details of arc voltage and current voltage for a $100 \mu \mathrm{m}$ gap.

The same experiment was performed with three inter-electrode gaps, of 10, 100 and $1000 \mu \mathrm{m}$ and two current values $\left(1 \mathrm{~A}_{\mathrm{rms}}\right.$ and $\left.4 \mathrm{~A}_{\mathrm{rms}}\right)$ in order to determine how these parameters could affect the ability of the arc to reignite. The results are summarized in table I. Three behaviors were seen. The first was when the arc could reignite at each half-cycle. The inter-electrode gas was hot enough to allow the production of plasma when the voltage reached $20 \mathrm{~V}$ (the threshold for arc ignition in carbon [9]). The second case was when the circuit remained closed after some arcing had taken place. This is due 
to the ejection of particles by the arc plasma that for a small gap $(10 \mu \mathrm{m})$ could close the circuit and allow the current to pass without creating an arc. The third case was when the current, relative to the gap, was not sufficient to promote the plasma. The current is the crucial parameter here for since for a given material, the arc voltage is practically constant at a fixed value (20-25V for the case of carbon, fig. 3$)$ the energy available to maintain the arc is determined by the supply current.

Table I: Arc behavior vs. inter-electrode gap and current.

\begin{tabular}{cccc}
\hline & $10 \mu \mathrm{m}$ & $100 \mu \mathrm{m}$ & $1000 \mu \mathrm{m}$ \\
\hline $1 \mathrm{~A}$ & arcs & extinction & extinction \\
& & & \\
$4 \mathrm{~A}$ & closed circuit & arcs & extinction \\
\hline
\end{tabular}

\subsection{AC Discharge Arc}

For small distances between the electrodes and high voltage, the arc can start by breakdown in the inter-electrode gap. To characterize this effect, the procedure for initiating the arc was changed. The tests were performed with a supply voltage of $220 \mathrm{~V}$, $50 \mathrm{~Hz}$ and a current of 2 to $4 \mathrm{~A}$, but the voltage was applied after the movement had reached the chosen gap. Inter-electrode gaps from $0 \mu \mathrm{m}$ to a few hundred microns were studied. For each test, the ignition voltage of the first arc (that is the measured inter-electrode voltage at which an arc appears), the sustained arc voltage and the persistence of the arcs during the time that the current was supplied (about 2 s) were observed. As before, one of the electrodes was rounded, the other flat and they were cleaned after each test.

The first test was performed with porous graphite electrodes. Ignition voltages of the first arc as a function of inter-electrode distance for two currents, $2 \mathrm{~A}$ and $4 \mathrm{~A}$ are 
presented in fig. 4. If the arcs persisted for about $2 \mathrm{~s}$, the points are full, otherwise, if at any time while the current was supplied, the arcs ceased permanently to re-ignite, the data points are open.

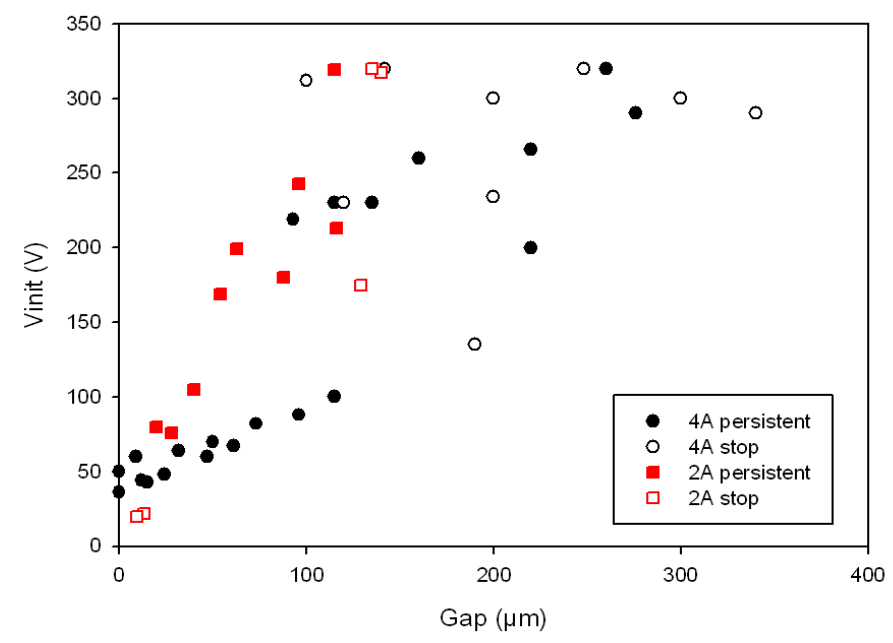

Fig. 4 Arc ignition voltage vs. inter-electrode gap for porous graphite electrodes. Solid points indicate where an arc is sustained while open points are for measurements where the arc extinguished.

The first piece of information to be gathered from this curve is that arcs may be started for voltages well below the theoretical Paschen voltage (the minimum breakdown voltage between smooth electrodes at atmospheric pressure is about $330 \mathrm{~V}$ for a distance of $8 \mu \mathrm{m}$; it is higher for shorter and longer distances). This phenomenon has been observed for the case of metal contacts where experiments [10-12] have shown the existence of arcs for gaps between electrodes less than $10 \mu \mathrm{m}$ with ignition voltages proportional to the distance. This is explained by the vaporization of rough asperities on the surface that initiate a glow discharge and which, due to the passage of current, are vaporized due to heating by the Joule effect. This vaporization phenomenon is similar to a molten bridge explosion and allows the arc to start [12]. The data shown in 
Fig. 4 indicates that arcs between graphite electrodes can occur with much greater distances $(100 \mathrm{~s}$ of $\mu \mathrm{m})$ for a given voltage compared to what has been seen for metal electrodes (up to $10 \mu \mathrm{m}$ for voltages below the Paschen voltage) [12]. In addition, an effect of the supply current is visible (a higher current facilitates the emergence of an arc at a given distance, that is to say that the voltage necessary to achieve ignition is less). For this type of material, arcs can appear up to a distance between electrodes of $350 \mu \mathrm{m}$ at an arc current of $4 \mathrm{~A}$. Beyond $300 \mu \mathrm{m}$, they cannot persist. At a lower supply current of $2 \mathrm{~A}$, there is no appearance of an arc for distances greater than $150 \mu \mathrm{m}$.

The same experiment was repeated with a supply current of $4 \mathrm{~A}$ with electrodes made of dense carbon (POCOGRAPHITE EDM-1, avg. Particle size $<5 \mu \mathrm{m}$ ) and copper with the following pairings: $\mathrm{C}-\mathrm{C}$ dense, $\mathrm{Cu}-\mathrm{Cu}, \mathrm{Cu}$ (cathode) $-\mathrm{C}, \mathrm{Cu}-\mathrm{C}$ (cathode). The term cathode refers to the electrode at the first current half-cycle. These results are presented in fig. 5 .

The results for electrodes made of dense graphite are different from those for porous graphite. In this case there is no appearance of arcs for distances greater than $30 \mu \mathrm{m}$ for sub-Paschen voltages. This seems to be explained by the fact that the dense material is less rough than the porous material. If the occurrence of arcs is due to a rough surface effect, then smooth electrodes do not favor the appearance of arcs. This shows the importance of material structure on these properties related to the ignition of arcs and their persistence.

For the case of copper electrodes, arcs appeared but never persisted over more than a few half-cycles of the current. Thus we have seen how porous graphite significantly facilitates the emergence and persistence of arcs. 


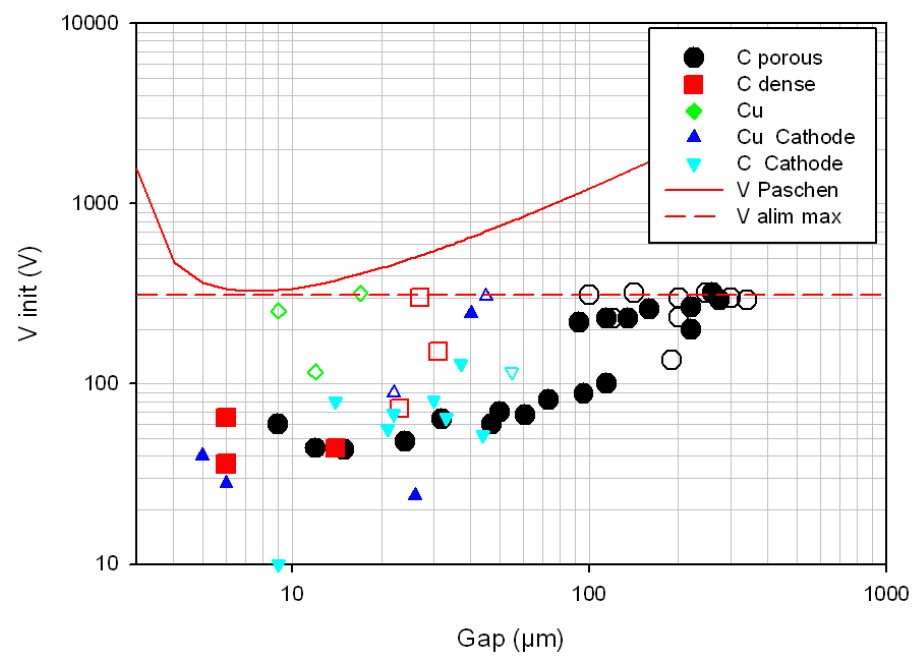

Fig. 5 Arc voltage ignition vs. inter-electrode gap. Also shown is the theoretical

Paschen curve for breakdown in air. Open points indicate that the arcs did not persist.The triangles refer to measurements where one electrode was copper and the other porous carbon, as indicated.

Fig. 7 also presents the case when the first arc appears for a copper cathode (rounded) and a dense graphite (flat) anode. Even with a copper cathode, a case which should have been similar to that of copper - copper, arcs appear for distances greater than $20 \mu \mathrm{m}$. Moreover, the persistence of arcs was facilitated, this probably due to the effect of free carbon which participates in the arcs that follow. The reversal of the electrodes is also shown with the same gaps being used. However, the voltages were lower. Having a carbon electrode makes it much easier for the emergence of the first arc at a given voltage level. For arcs that follow, their re-ignitions depend mostly on the plasma state and the inter-electrode distance.

In conclusion, the most suitable material to produce and sustain arcs is porous graphite followed by dense graphite. C cathode - $\mathrm{Cu}$ anode is next and only $\mathrm{Cu}-\mathrm{Cu}$ does not present this behavior $[13,14]$.

Even if arcs due to different electrodes present different behaviors (in terms of 
ignition voltage for example), in the case of $\mathrm{Cu}-\mathrm{C}$, the graphite electrode tends to deposit carbon on the copper electrode (see photographs below) $[13,14]$. The principle consequence is that, after a few arcs, the arcing behavior becomes like that for the $\mathrm{C}-\mathrm{C}$ case, as we can see in fig. 6.

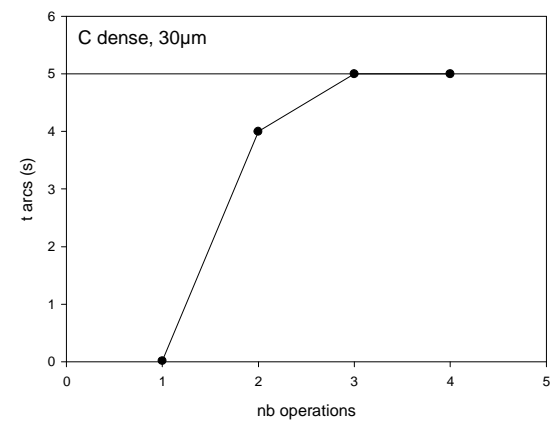

Fig. 6 Total arc duration for a copper-carbon electrode pair and an inter-electrode gap of $30 \mu \mathrm{m}$ compared to that for dense carbon electrodes with the same gap.

This figure presents the ability of the arc to reignite (i.e. to sustain an arc voltage over a period of 5s) vs. the number of operations. The procedure was the same as in the previous tests with an inter-electrode gap of $30 \mu \mathrm{m}$ but without the electrodes being cleaned before each test. As shown, for the two first operations, the arcing time (that includes re-ignition) is less than $5 \mathrm{~s}$ as the arcs self-extinguish. After the third operation, arcs no longer self-extinguish. The behavior tends towards that seen for C-C electrodes. Indeed, as shown in fig. 7, a carbon layer is deposited on the copper electrode and finally presents the same behavior as that for a graphite electrode pair.

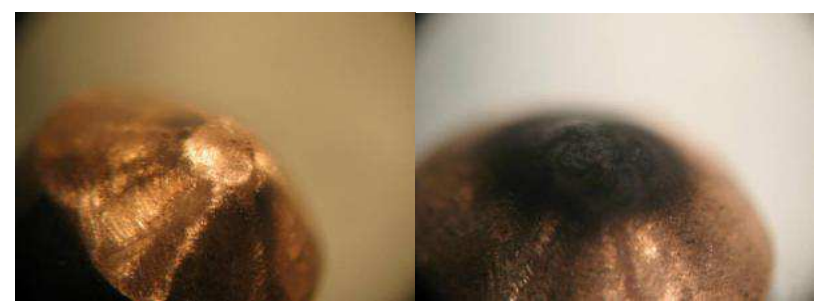


Fig. 7 Copper cathode before and after arcing.

\subsection{X-Ray Scattering experiments}

With the goal of understanding carbon arcs in more detail, we have used $x$-rays to explore the character of nanoparticles formed in arcs between porous carbon electrodes. The Small Angle X-Ray Scattering method was used for these studies. The SAXS technique involves passing a high energy $x$-ray beam through a sample (in this case the inter-electrode gap) and registering the scattered x-rays using a 2-D detector. We have used SAXS recently to investigate particles formed in arcs between silver-tin-oxide electrodes [15]. It is a very powerful technique since, unlike optical scattering methods, knowledge of the index of refraction of the particles is not required. This is important as this is a parameter that can change with the particle temperature which in this case will be very high. Given the dilute nature of the samples, high energy $x$-ray beams produced by $3^{\text {rd }}$ generation synchrotron radiation sources such as the Soleil synchrotron are needed to provide sufficient signal strength for accurate measurements. 


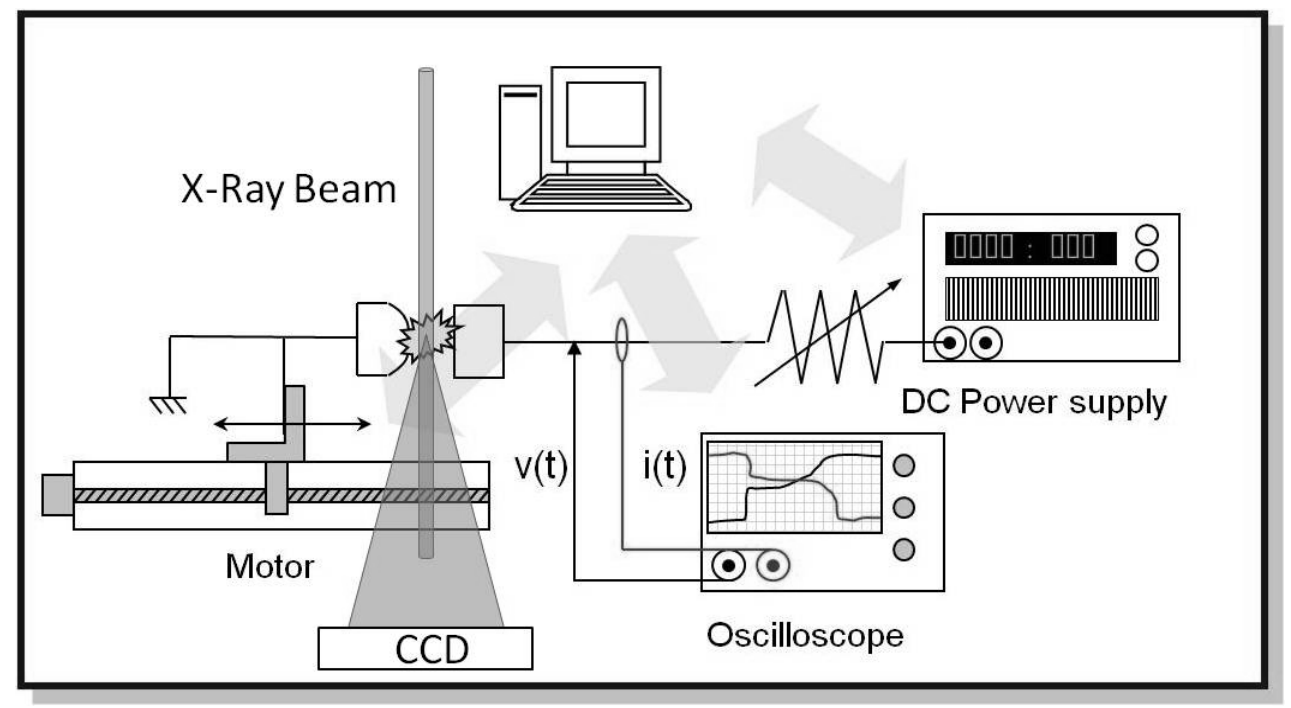

Fig. 8: Schematic of the experimental test apparatus showing the $x$-ray beam passing between the electrodes and being captured on a CCD detector. The measurement and control system is also schematically presented.

The experimental apparatus (fig. 8) is the same as that used in the other tests described above and the whole assembly was mounted on the SWING beamline [16] at the Soleil synchrotron. A voltage/current pair of $200 \mathrm{~V}$ and $8 \mathrm{~A}$ was chosen in order to maintain a stable arc with a large electrode $(2 \mathrm{~mm})$ gap during the X-ray exposure.

\section{Results and Discussion}

Energy selected (12 keV) X-rays exited the synchrotron beamline through a 10 micron thick mica window, passed through the electrode region (in air) and through a second mica window into a vacuum tank containing a $1024 \times 1024$ pixel $x$-ray detector. The total air path was $15 \mathrm{~cm}$ long to allow sufficient distance between the arc region and the windows to avoid any heat damage or particle deposition on the window surfaces. 
The scattering is parameterized by the magnitude of the momentum transfer vector $\mathrm{q}=$ $(4 \pi / \lambda) \sin \theta / 2$ where $\lambda$ is the wavelength of the $\mathrm{X}$-rays and $\theta$, the scattering angle around the primary beam direction. The movable detector was positioned at a distance of 2 meters from the sample providing a q range of $0.0045-0.54 \AA^{-1}$. However, due to air scattering and detector sensitivity, the useful upper value for q was about $0.05 \AA^{-1}$. The size $\mathrm{D}$ of the particles detected is of the order of $\pi / \mathrm{q}$ [17] so this means that our detection system was sensitive to particles in the size range from 4 to $70 \mathrm{~nm}$. In these experiments, the x-ray shutter was opened, the voltage was applied to the electrodes, the detection sequence of typically 20 frames each lasting $10 \mathrm{~ms}$ separated by $250 \mathrm{~ms}$, was initiated and then the voltage was cut off after 3 seconds while the detector continued to take data. At the end of this the $x$-ray shutter was closed. We did not see any effect of the $x$-ray beam on arc initiation and the arc extinguished as soon as the voltage was cut.

Graphs of Intensity versus q were plotted and curves taken at the end of this period, when the arc was extinguished, were subtracted from those indicating the presence of particle scattering to separate contributions due to air scattering.

\begin{tabular}{|c|c|c|}
\hline \begin{tabular}{|c|c|}
\hline Fig. 9a: X-ray scattering data \\
plotted as Intensity vs q. The blue
\end{tabular} & Fig. 9b: (ibid.) but there is no \\
curve is a Guinier fit to the data at & fitting was performed. The Porodfitis \\
\hline
\end{tabular}




\begin{tabular}{|l|l|}
\hline low q while the green straight line is & shown (in green). \\
a power law fit to the data at high q. & \\
The red curve through the data points & \\
is an overall fit to the data using the & \\
macro in [21]. &
\end{tabular}

It was found that particle scattering signals on top of a background due to air scattering were seen during some of the initial exposures but after the arc extinguished, these disappeared into the background. Thus signals due to particle scattering alone were obtained by subtracting the scattering curves of later exposures from those showing the presence of particles in the early exposures while the arc was lit. Something that was surprising was that particles were not always evident in the initial exposures but appeared after the third or fourth or in one case, in the $9^{\text {th }}$ exposure. An arc is an intensely hot and violently turbulent region and the abrupt disappearance of particle signals would seem to reflect their rapid dispersion in this turbulent field. Alternatively, the rapid disappearance of the particles may be due to attack by atmospheric oxygen leading to their conversion to gaseous carbon oxides. The arc is generated from arc spots on the electrodes (generally the cathode) that move around very rapidly $[13,14,18]$ and so even though the beam passes through the geometrical center of the inter-electrode gap, at a given instant, the arc may be offset from this so that the beam may be interrogating a cooler region where particle condensation can take place. (Indeed this can be seen in video 3 of the supplemental material). At other times, the $\mathrm{x}$-rays pass through the hot arc where solid particles cannot exist. This can be the explanation for the rather chaotic nature of the observations. 
Figs. $9 \mathrm{a}$ and $9 \mathrm{~b}$ show two sets of scattering data taken with the $\mathrm{x}$-ray beam centered between the electrodes. The curves of I(q) vs q generally exhibit two behaviors referred to as the Guinier region (at low q) and the Porod (high q) region [19]. In the Guinier region, the intensity can be fitted to the expression:

$$
I(q)=G \exp \left(-q^{2} R_{G}{ }^{2} / 3\right)
$$

where $\mathrm{R}_{\mathrm{G}}$ is the radius of gyration of the particles and $G=N \rho^{2} V^{2}$ with $N$, the particle number density, $\rho$, the scattering length and $V$, the particle volume. In the Porod region:

$$
\mathrm{I} \approx \mathrm{q}^{-\mathrm{p}}
$$

If $p=4$, the surface of the particles is smooth, if $p=3$, the surface is rough and this can indicate an aggregated character. If $p=2$, the particles form a Gaussian chain [20]. Analysis of the scattering data shown in the figures has been performed using the Irena package of Macros developed by Jan Ilavski of the Advanced Photon Source [21]

From fig. 9a it is seen that the Guinier fit is just possible and in this case yields a value of $R_{G}=31 \mathrm{~nm}$ for the mean particle size. In fig. $9 \mathrm{~b}$ however, it is clear that there is no apparent Guinier region within our q range. This indicates that the particles doing the scattering are large $\left(R_{G}>78 \mathrm{~nm}\right)$. Over all the measurements taken with porous carbon electrodes, the Guinier region was either absent or the complete region could not be seen within our q range. This was in strict contrast to the case of arcs between silver-tin-oxide electrodes [15] where it was found that nanoparticles thus formed were in the rather narrow size range from 30-40 $\mathrm{nm}$.

What was most interesting about these observations was that unlike the silver-tin-oxide case where the particles were found to have smooth surfaces [15], in the case of carbon electrodes the I(q) vs q curves displayed a slope of about 3.2 for all the observations made. This indicates that their surface is rough. In other experiments that 
we have performed using SAXS, on soot nanoparticles in flames [22,23] or on copper nanoparticles in a microwave discharge in air [24], it was found that there were several families of particles with smooth sub-primaries and aggregated primary particles. (At least in the soot case, these primaries generally are further aggregated into larger fractal structures whose size falls outside the q range of our measurements). For the silver-tin-oxide case, only a single family was seen suggesting that these particles are formed through nucleation and coalescence in the liquid state, thus displaying a smooth surface. In the case of the carbon electrodes, again there is no indication of more than one family of particles observable in our q range. The fact that the surface is rough suggests that they are formed by nucleation and surface condensation. At atmospheric pressure there is debate as to whether carbon has a liquid phase but if so its melting point is between 3800 [25] and $5000^{\circ} \mathrm{K}$ [26,27]. This can explain the different morphology of these particles as their condensation and growth will take place in the solid phase.

Significantly efforts to analyse particles formed between dense carbon electrodes proved to be unsuccessful even though the electrical conditions and inter-electrode gap were the same as for the porous carbon case. Most likely the reason for this failure was that fewer particles were formed so that the resulting scattering signals were too weak to rise above the background noise.

\section{Discussion}

The observation that is most striking from these measurements is the ease of generating a strong electrical arc using graphite electrodes particularly when the graphite is porous. This has been attributed to the increased roughness of the surface compared with a 
metallic electrode. Electrical breakdown during contact breaking with metallic electrodes passes through a molten bridge phase when at the final point of contact, a high current passes between microscopic asperities on the contact surfaces and this molten bridge explodes generating an ionized plasma and initiating the arcing process [9]. In the breaking experiments reported here, a similar phenomenon will occur except that there is no molten phase for carbon and the electron generation is produced by the explosion of asperities due to local high electric fields. This is also a violent process at the current densities experienced here and leads to the formation of craters on the electrode surfaces. The sharp edges of these craters are excellent sites for further breakdown phenomena $[13,14,18]$ that will sustain arcing during the alternating current cycles.

When considering the explosive release of electrons from rough asperities on the surface of the material, a key parameter is the rate at which energy can be supplied to the material so that it does not have time to adjust and to conduct away this energy input [28]. This phenomenon was first recognized by Mesyats [29] who developed the "Ecton" (explosion center) model to describe it. If the rate of specific energy input $\mathrm{dw} / \mathrm{dt}$ is greater than the maximum rate of heat removal, then the asperity will explode. One can introduce the delay time $\tau_{d}$ for this explosion and this can be related to the current density by the expression:

$$
\int_{0}^{t_{d}} j^{2} d t=\bar{h}
$$

where $\bar{h}$ is called the specific action and depends upon the surface material. For carbon, this has a value of $1.8 \mathrm{~A}^{2} \mathrm{~s} / \mathrm{m}^{4}$ while that for copper is $41 \mathrm{~A}^{2} \mathrm{~s} / \mathrm{m}^{4}[13,14,18]$. Thus for the same current density, the time for the explosion of an asperity will be 22 
times faster for carbon than for copper. This is due to the greater thermal conductivity of copper that removes energy faster from the asperity. Another feature that must be taken into account is not only the initial presence of asperities but also the subsequent formation of asperities caused by arcing. For metal electrodes, the formation process for asperities is via a combination of melting and local high electric field that produces cone-shaped micro-protrusions that subsequently explode due to the high current density. As seen by the video 3 in the supplemental material, the surface of the carbon electrodes is constantly eroded by the arcs leading to ablative roughening.

The violence of the arc can also lead to the release of microscopic chunks of carbon that for small gaps, can act as a bridge between the electrodes. This can be seen in the slow motion video of a carbon arc included in the supplemental material to this article (video3). Figures $10 \mathrm{a}$ and $10 \mathrm{~b}$ show two snapshots taken from this video and the presence of hot carbon bridges and free macroscopic carbon particles can be seen.

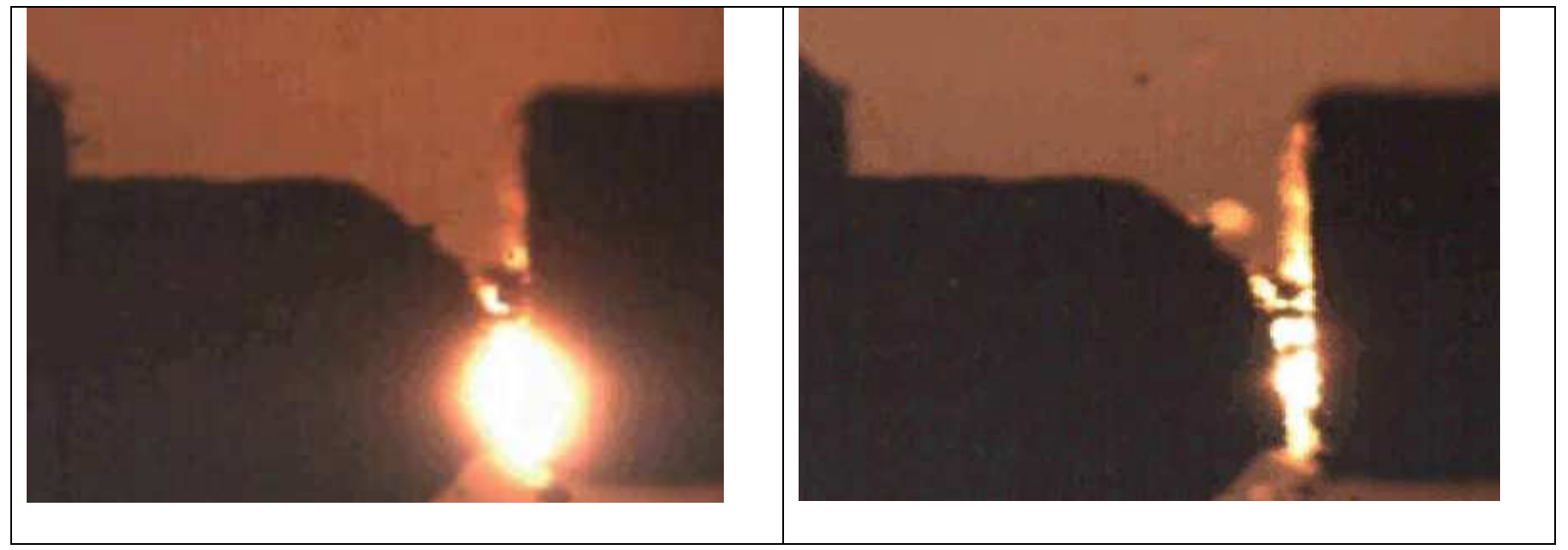

Figs. $10 \mathrm{a}$ and $10 \mathrm{~b}$. Still photographs taken from video 3 in the supplemental material showing the presence of solid carbon bridges formed by arc damage to the electrodes that present excellent triggers for further arcing. 
Another feature of the carbon arc which can play a role in sustaining it is the fact that the particles formed in the arc condense to the solid form and being very hot are able to undergo thermionic emission releasing electrons that can be accelerated by the alternating field causing re-ignition of the plasma. They can also be reservoirs for electrical charge due to the attachment of free electrons to cooler particles. Although it is not possible to measure this directly in these experiments, we can take as an analogy a hydrocarbon flame where extensive research has shown that soot particles are known to be electrically charged and usually there is an excess of positive charge on these particles [30]. Thermionic emission is one of the major ionization mechanisms for the charging of soot particles. Carbon has a very high vaporization point $\left(4827^{\circ} \mathrm{C}\right)$ and so these particles make excellent thermionic emitters over a wide energy range as they condense out from the hot plasma arc. The work function for amorphous carbon is about $4.9 \mathrm{eV}$ [31] while that for carbon nanotubes is 4.5-4.6 eV [32]. Copper has a melting point of $1083^{\circ} \mathrm{C}$ and a boiling point of $2567^{\circ} \mathrm{C}$ and so it is much more limited as a thermionic emitter over the temperature range of the arc. Another factor that may play a role in electron generation is the fact that the rough surfaces noted in the above measurements are conducive to the phenomenon of field emission. Thus high electrical fields can be generated around the asperities on the particle surfaces that are capable of ripping electrons out from the surface $[33,34]$. This phenomenon is well known for carbon nanotubes and has been discussed with respect to electrical arcing in ref. [18]. Further investigation of this proposed mechanism for electron production will involve new SAXS measurements exploring the lower $q$ range to determine the exact size and number density of the carbon particles that we have shown to be present in the 
inter-electrode space.

Another aspect of sustainability of arc is the arc voltage or burning voltage i.e. the voltage necessary for maintaining an arc lit $[13,14,18]$. Many workers have sought correlations between this voltage and physical parameters of the electrode materials. In particular a relationship has been found between the cohesive energy (the energy necessary to free an electrically neutral atom from a solid at $0^{\circ} \mathrm{K}$ ) which for carbon is $7.37 \mathrm{eV} /$ atom and for copper $3.49 \mathrm{eV} /$ atom and the burning voltage. This suggests that the voltage required for carbon is greater than that for copper. Anders et al. [35] found a value of $29.6 \mathrm{~V}$ at 300 A for a vacuum arc between carbon electrodes compared to 23.4 $\mathrm{V}$ for copper electrodes. Other workers have found lesser values (16-19 V) for carbon $[13,14]$. As seen in figure 3 , the measured arc voltage ranged from 20 to $25 \mathrm{~V}$ in a given cycle with porous carbon electrodes. In the case of copper electrodes in air at atmospheric pressure, we found that arcs did not restrike and persist (fig. 5). This suggests that an initial asperity exploded but new ones were not formed. For the case of the mixed copper-carbon electrode pair, as noted above, after a few cycles this became like a carbon-carbon pair due to the deposit of a surface layer of carbon on the copper electrode.

\section{Conclusion}

This work has presented the characteristics of arcs similar to series arc faults and their enhanced capability of ignition and re-ignition when the electrodes are made of porous carbon. This result has direct relevance to electrical safety as it is clear that a degraded carbonized environment within cable insulation, caused by sparking, 
enhances the capability of arcs to re-ignite that could lead to fire [1-3]. In the supplemental material to this article, there is a slow-motion video (video 2) taken for a cable, previously damaged by sparking that has been sectioned and the end glued to a glass plate so that the arcing could be viewed directly. It is seen that at every $50 \mathrm{~Hz}$ current cycle, either arcing occurs or islands (fig.2) of pulsating glowing material are seen that generate a violent re-ignition of the arcing. The hot carbon particles are clearly implicated in the development of the arc plasma. As seen in the first video in the supplemental material, this arcing leads to the eruption of a jet of flame as flammable vapors resulting from the breakdown of the polymer insulation, break free from the surface (fig.1). It is not difficult to imagine what the consequences may be if this cable were to be in contact with flammable material during such an event. In work to be published elsewhere [36], we have examined this cable burning problem using x-ray and electron-microscopic techniques to characterize the impact on the cable material and structural and chemical changes due to the presence of electrical arcing.

\section{Acknowledgements}

The authors would like to acknowledge the financial and technical assistance of Schneider Electric for this work. They would also like to thank Synchrotron Soleil and its staff for the financial and technical support of the $x$-ray scattering measurements.

\section{References}

[1] J.J. Shea, Fire and Materials, 2010;35: 19-42

[2] J.J. Shea, 51 ${ }^{\text {st }}$ IEEE Holm Conf. on EC, 2005, pp 167-175 
[3] J. A. Wafer 51 ${ }^{\text {st }}$ IEEE Holm Conf. on EC, 2005, pp156-16

[4] C. E. Restrepo, "Arc fault detection and discrimination methods", Proc. IEEE Holm Conf. on EC, 2007, pp. 115-122.

[5] D. Kolker, S. Campolo, N. DiSalvo, "A study of time/current characteristics of the ignition processes in cellulosic material caused by electrical arcing for application in 240v arc-fault circuit interrupters" Proc. IEEE Holm Conf. on EC, 2007, pp. 105-114.

[6] E. Carvou, N. Ben Jemaa, "Experimental Studies on Arc Duration in Ac Voltage Supply" Proc. of the 24th International Conference on Electrical Contacts; St Malo (France), pp 7-12, 2008.

[7] J. Shea, "Comparing 240 Vrms to 120 Vrms Series Arcing Faults in Residential Wire", Proc. IEEE Holm Conf. on EC, 2008, pp. 218-224.

[8] E. Carvou, N. Ben Jemaa, "Electrical arc study in the range of 14-112vdc for automotive power contacts" Proc. of the 23rd International Conference on Electrical Contacts; Sendai (Japan), 2006, pp 28-33.

[9] Slade "Electrical contacts, Principles and Applications”, Marcel Dekker, Inc., New York, 1999, p1025.

[10] R.T. Lee, H.H. Chung and Y.C. Chou, IEE Proc - Sci. Meas. Technol. 2001;148: 8. [11] J-M Torres and R S Dhariwal Nanotechnology 1999; 10: 102.

[12] Paul G. Slade, Erik D. Taylor, "Electrical Breakdown in Atmospheric Air Between Closely Spaced (0.2 $\mu \mathrm{m}-40 \mu \mathrm{m})$ Contacts, Proc. IEEE Holm Conf., 2001, pp. 245-250. [13] J.J. Shea and X. Zhou, $25^{\text {th }}$ International Conference on Electrical Contacts and 56 ${ }^{\text {st }}$ IEEE Holm Conf on EC, 2010, pp 188-198

[14] J.J. Shea and J.B. Carrodus, 57 ${ }^{\text {th }}$ IEEE Holm Conf. on EC, 2011, pp 129-137

[15] E. Carvou, J.L. Le Garrec, J. Pérez, J. Praquin, M. Djeddi and J.B.A. Mitchell, AIP Advances 2013;3:032139 
[16] http://www.synchrotron-soleil.fr/Recherche/LignesLumiere/SWING

[17] A. Guinier, X-Ray Diffraction in Crystals, Imperfect Crystals and Amorphous Bodies, Dover Publications, Inc, New York, 1994.

[18] A. Anders, Cathodic Arcs, New York: Springer, 2008.

[19] D.I. Svergun, A.V. Semenyuk and L.A. Feigin Acta. Cryst., 1988; A44: 244.

[20] G. Beaucage, Phys. Rev. E, 2004; 70: 031401.

[21] J. Ilavsky, J. Appl. Cryst., 2009; 42: 347.

[22] J. B. A. Mitchell, S. di Stasio, J.L. LeGarrec, A.I. Florescu-Mitchell, T. Narayanan and M. Sztucki, J. Appl. Phys., 2009; 105: 124904.

[23] J. B. A. Mitchell, J. Courbe, A.I. Florescu-Mitchell, S. di Stasio, and T. Weiss, J. Appl. Phys., 2006; 100: 124918.

[24] E. Jerby, A. Golts, Y. Shamir, S. Wonde, J.B.A. Mitchell, J.L. LeGarrec, T. Narayanan, M. Sztucki, D. Ashkenazi, Z. Barkay and N. Eliaz, Appl. Phys. Lett., 2009; 95: 191501 .

[25] A.G. Whitaker and P.L. Kintner, Carbon, 1985; 23: 255.

[26] E.I. Asinovskii, V.M. Batenin, I.I. Klimovskii and V.V. Markovets, High Temp., 2001; 39: 739 .

[27]D.V. Abramov, S.M. Arakelyan, A.F. Galkin, L.D. Kvacheva, I.I. Klimovskii, M.A. Kononov, L.A. Mikhalitsyn, A.O. Kucherik, V.G. Prokoshev and V.V. Savranskii, JETP Letters, 2006; 84: 258 .

[28] A.B. Fialkov, Prog. Energy Combust. Sci. 1997; 23: 399.

[29] A. Ilie, A. Hart, A.J. Flewitt, J. Roberston and W.I. Milne, J. App. Phys., 2000; 88: 6002.

[30]P. Liu, Y. Wei, K. Jiang, Q. Sun, X. Zhang, S. Fan, S. Zhang, C. Ning and J. Deng, 
Phys. Rev. B, 2006; 73: 235412.

[31] W.C. Chace and M.A. Levine J. App. Phys. 1960; 31: 1298.

[32] G.A. Mesyats, IEEE Transactions on Plasma Sci., 1995 23: 879.

[33] Y. Saito, K. Hamaguchi, T. Nishino, K. Hata, K. Tohji, A. Kasuya and Y., Jap. J. Appl. Phys. 1997; 36: L1340[33]

[34] J-M Bonard, T. Stockli, F. Maier, W.A de Heer, A. Chatelain, J-P. Salvetat and L. Forro, Phys. Rev. Lett. 1998; 81: 1441.

[35] A. Anders, B. Yotsombat and R. Binder, J. Appl. Phys., 2001; 89: 7764.

[36] E. Carvou, E. Yin Kee Choi, J.L. Le Garrec and J.B.A. Mitchell (In preparation) 